\title{
Trigonometric Function Solutions of Fractional Drinfeld's Sokolov -Wilson System
}

\author{
Zeynep Fidan Koçak $^{1}$ and Gülnur Yel $^{2, *}$ \\ ${ }^{1}$ Department of Mathematics, University of Mugla Sitkı Koçman, Mugla, Turkey \\ ${ }^{2}$ Department of Mathematics Education, Final International University, Kyrenia, Cyprus
}

\begin{abstract}
In this paper, we construct exact trigonometric solutions of the space-time fractional classical Drinfeld's Sokolov-Wilson system by Modified Trial Equation Method (MTEM). These solutions may explain some physical phenomena and lead to researchers in physics and engineering.
\end{abstract}

\section{Introduction}

Studies in recent years on fractional analysis have been increasingly proceed. Fractional analysis describe many scientific phenomena such as physics, chemistry, biology, engineering and so on. Several efficient analytical, semi-analytical and numerical methods are applied for solving fractional equations and equation systems. Some of them are Modified Trial Equation method, Sumudu Transform method, Riccati-Bernoulli sub-ODE method, İmproved Bernoulli sub-equation method, modified Kudryashov method, Functional Variable method, Homotopy Analysis method and so on have been used to find new solutions of fractional equations [1-11]

We apply Modified Trial Equation method (MTEM) for obtain new travelling wave solution to fractional Drinfeld's Sokolov-Wilson system $[1,12]$.

\section{The Modified Trial Equation Method}

In this subsection we describe structure of the MTEM [2-5].

We consider partial differential equation in two variables and a dependent variable $u$ :

$$
P\left(u, u_{t}, u_{x}, u_{x x}, u_{x x x}, \ldots\right)=0,
$$

and take the wave transformation,

$$
u(x, t)=u(\xi), \xi=k x-c t,
$$

where $k$ and $c$ are constants can be determined later. By substituting Eq. (2.2) into Eq. (2.1), a nonlinear ordinary differential equation (NODE) is converted as following:

$$
N\left(U, U^{\prime}, U^{\prime \prime}, U^{\prime \prime \prime}, \ldots\right)=0 .
$$

We assume that the solution can be expressed in the form

$$
\begin{gathered}
U^{\prime}=\frac{F(u)}{G(u)}=\frac{\sum_{i=0}^{n} a_{i} u^{i}}{\sum_{j=0}^{l} b_{j} u^{j}}=\frac{a_{0}+a_{1} u+a_{2} u^{2}+\cdots+a_{n} u^{n}}{b_{0}+b_{1} u+b_{2} u^{2}+\cdots+b_{l} u^{l}}, \\
U^{\prime \prime}=\frac{F(u)\left(F^{\prime}(u) G(u)-F(u) G^{\prime}(u)\right)}{G^{3}(u)}
\end{gathered}
$$

where $F(u)$ and $G(u)$ are polynomials. Substituting above relations into Eq.(2.3) yields an equation of polynomial $\Omega(u)$ of $u$ :

$$
\Omega(u)=\rho_{s} u^{s}+\cdots+\rho_{1} u+\rho_{0}=0 .
$$

According to the balance principle, we can get a relationship between $n$ and $l$. We can compute some values of $n$ and $l$.

Let the coefficients of $\Omega(u)$ all be zero will yield an algebraic equations system:

$$
\rho_{i}=0, i=0,1,2, \cdots, s .
$$

By solving this system, we will thus determine the values of $a_{0}, a_{1}, \ldots, a_{n}$ and $b_{0}, b_{1}, \ldots, b_{l}$.

Reduce Eq.(2.4) to the elementary integral form,

$$
\pm\left(\mu-\mu_{0}\right)=\int \frac{G(u)}{F(u)} d u .
$$

\footnotetext{
*Corresponding author: gulnuryel33@gmail.com
} 
Using a complete discrimination system for polynomial of $F(u)$, we solve Eq.(2.8) with the help of Mathematica 9 and classify the exact solutions to Eq.(2.3). For better explication of results obtained in this way, we can plot two and three dimensional surfaces of the solutions obtained by using suitable parameters.

\section{Application}

Let consider the the space-time fractional classical Drinfeld's Sokolov-Wilson system as follows [1],

$$
\begin{aligned}
& \frac{\partial^{\alpha} u}{\partial t^{\alpha}}+p v\left(\frac{\partial^{\beta} v}{\partial x^{\beta}}\right)=0 \\
& \frac{\partial^{\alpha} v}{\partial t^{\alpha}}+q\left(\frac{\partial^{3 \beta} v}{\partial x^{3 \beta}}\right)+r u\left(\frac{\partial^{\beta} v}{\partial x^{\beta}}\right)+s v\left(\frac{\partial^{\beta} u}{\partial x^{\beta}}\right)=0
\end{aligned}
$$

where $u$ and $v$ are the functions of $(x, t)$, $0<\alpha, \beta \leq 1, x>0$. We apply following transformations then the Eq. (3.1) can be reduced to ordinary differential equation.

$$
\begin{aligned}
& u(x, t)=U(\eta), v(x, t)=V(\eta), \\
& \eta=\frac{w x^{\beta}}{\Gamma(1+\beta)}-\frac{\lambda t^{\alpha}}{\Gamma(1+\alpha)},
\end{aligned}
$$

where $w$ and $\lambda$ are arbitrary constants and $a \neq 1, b \neq 1$ are fractal constants. We get differentiate the function $U(\eta), V(\eta)$ respect to $\eta$, then Eq.(3.2) is rearranged, it yields us,

$$
\begin{gathered}
-\lambda b U^{\prime}+p a w V V^{\prime}=0 \\
-\lambda b V^{\prime}+q a^{3} w^{3} V^{\prime \prime \prime}+\operatorname{raw} U V^{\prime}+\operatorname{saw} V U^{\prime}=0 .
\end{gathered}
$$

We obtain following equalities from Eq.(3.3),

$$
\begin{aligned}
& U^{\prime}=\left(\frac{p a w}{b \lambda}\right) V V^{\prime}, \\
& U=\left(\frac{p a w}{b \lambda}\right) V^{2}+c_{1} .
\end{aligned}
$$

where $c_{1}$ is constant of integration. Embedding Eqs.(3.5,3.6) into Eq.(3.4), we have the nonlinear ordinary differential equation:

$$
\begin{aligned}
& q a^{3} w^{3} V^{\prime \prime \prime}+p a^{2} w^{2}\left(\frac{r+2 s}{2 b \lambda}\right) V^{2} V^{\prime}+ \\
& \left(r a w c_{1}-b \lambda\right) V^{\prime}=0
\end{aligned}
$$

Integrating Eq.(3.7) once,

$$
\begin{aligned}
& q a^{3} w^{3} V^{\prime \prime}+p a^{2} w^{2}\left(\frac{r+2 s}{6 b \lambda}\right) V^{3}+ \\
& \left(r a w c_{1}-b \lambda\right) V-c_{2}=0
\end{aligned}
$$

where $c_{1}$ is constant of integration. When we reconsider the Eq.(3.8) for homogenous balance principle between $V^{\prime \prime}$ and $V^{3}$, we obtain the following relationship for $n$ and $l$,

$$
2 n-2 l-1=3 \Rightarrow n=l+2,
$$

Case 1: For the values of $l=0, n=2$, we get ;

$$
\begin{aligned}
& V^{\prime}=\frac{F(v)}{G(v)}=\frac{\sum_{i=0}^{n} a_{i} v^{i}}{\sum_{j=0}^{l} b_{j} v^{j}}=\frac{a_{0}+a_{1} v+a_{2} v^{2}}{b_{0}}, \\
& V^{\prime \prime}=\frac{F(v)\left(F^{\prime}(v) G(v)-F(v) G^{\prime}(v)\right)}{G^{3}(v)} \\
& =\frac{\left(a_{1}+2 a_{2} v\right)\left(a_{0}+a_{1} v+a_{2} v^{2}\right)}{b_{0}^{2}},
\end{aligned}
$$

where $\quad a_{2} \neq 0$ and $b_{0} \neq 0$. Using the Wolfram Mathematica 9, the algebraic equation system is solved,

$$
\begin{aligned}
& a_{1}=0, c_{2}=0, r=\frac{\lambda b b_{0}^{2}-2 q a^{3} w^{3} a_{0} a_{2}}{a w b_{0}^{2} c_{1}}, \\
& p=-\frac{12 a^{2} b q w^{2} \lambda a_{2}^{2} c_{1}}{2 q a^{3} w^{3} a_{0} a_{2}-b_{0}^{2}\left(b \lambda+2 a s w c_{1}\right)},
\end{aligned}
$$

we have coefficients above. By substituting these coefficients in Eq.(3.8), we obtain the solution of $v(x, t)$ and $u(x, t)$

$$
v(x, t)=\frac{\sqrt{a_{0}} \operatorname{Tan}\left(\frac{\sqrt{a_{0} a_{2}}}{b_{0}}\left(\frac{w x^{\beta}}{\Gamma(1+\beta)}-\frac{\lambda t^{\alpha}}{\Gamma(1+\alpha)}\right)\right)}{\sqrt{a_{2}}},
$$

$$
u(x, t)=\frac{\operatorname{apwa}_{0} \operatorname{Tan}\left[\frac{\sqrt{a_{0} a_{2}}}{b_{0}}\left(\frac{w x^{\beta}}{\Gamma(1+\beta)}-\frac{\lambda t^{\alpha}}{\Gamma(1+\alpha)}\right)\right]^{2}}{2 b \lambda a_{2}}
$$




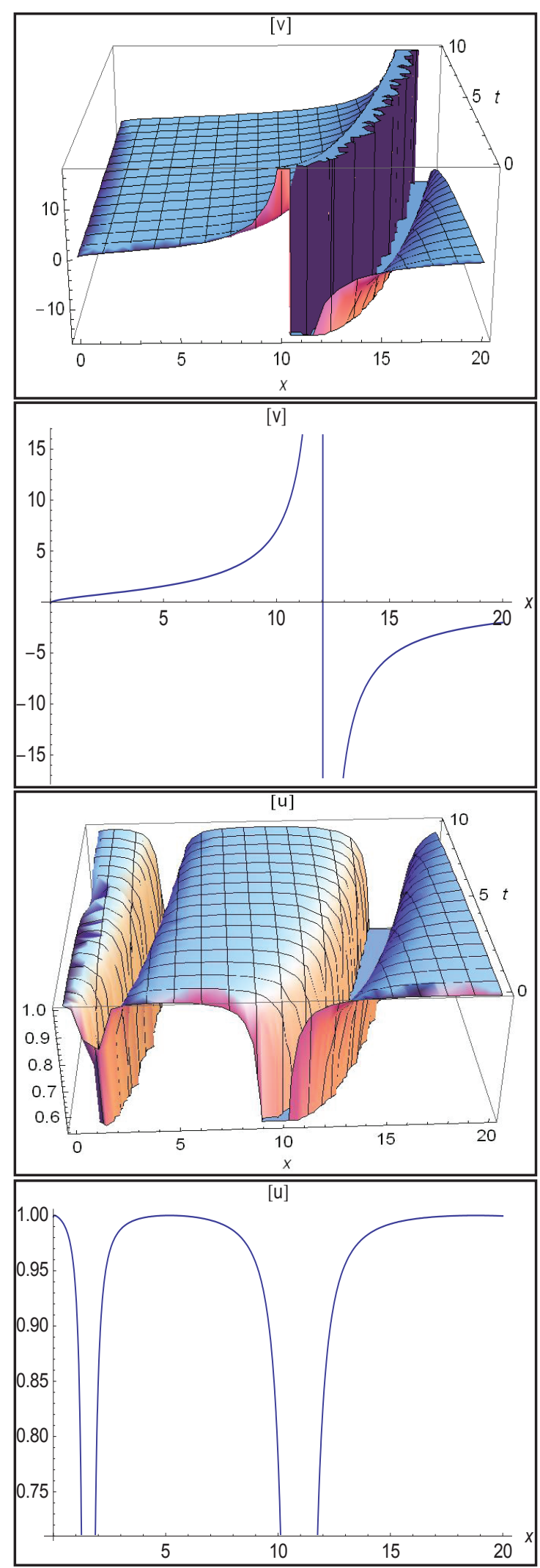

Fig. 1. The 2D and 3D surfaces for the the space-time fractional classical Drinfeld's Sokolov-Wilson system with $b_{0}=0.2, \lambda=0.3, a=b=0.1, \alpha=\beta=0.5$, $c_{1}=1, w=0.9, s=0.04,0<x<20,0<t<10$, $q=0.04, a_{0}=0.1, a_{2}=0.9$, and $t=0.4$, for 2D surfaces.

\section{Conclusions}

In this manuscript, we have efficiently and easily practice the Modified Trial Equation Method that is give analytical solution. We can say that this method is applied to nonlinear differential equations and systems. These trigonometric function solutions have been introduced to the literature for the first time. We think that these new solutions lead the way other scientific area.

\section{References}

1. A. R. Shehata, E. M. Kamal, and H. A. Kareem, Solutions of the space-Time fractional of some nonlinear systems of partial differential equations using modified Kudryashov method, IJPAM, 101(4), 477-487. (2015)

2. Z. F. Koçak, H. Bulut, and G. Yel, The Solution of Fractional Wave Equation by using Modified Trial Equation Method and Homotopy Analysis Method, AIP Conference Proceedings, 1637, 504. (2014)

3. H. Bulut, H. M. Baskonus, and Y. Pandir, The modified trial equation method for fractional wave equation and time fractional generalized Burgers equation, Abstract Appl. Anal., Article ID 636802, pp. 18. (2013)

4. H. Bulut, G. Yel, H. M. Baskonus, Novel Structure to the Coupled Nonlinear Maccari's System by Using Modified Trial Equation, Advanced Math. Models \& Applications, Vol.2, No.1, pp.14-19. (2017)

5. G. Yel, On The Analytical and Numerical Solutions of Nonlinear Fractional Differential Equations, Ph.D Thesis, Mugla Sitkı Koçman University, Mugla, 95 p. (2016)

6. H. Bulut, G. Yel, H. M. Baskonus, An Application of Improved Bernoulli SubEquation Function Method to The Nonlinear Time-Fractional Burgers Equation, Turk. J. Math. Comput. Sci. Vol.5,1-7. (2016)

7. H. Bulut, H. M. Baskonus and F.B.M. Belgacem, The Analytical Solutions of Some Fractional Ordinary Differential Equations By Sumudu Transform Method, Abstract Appl. Anal, Volume 2013, Article ID 203875, 6 pages. (2013)

9. S. T. Demiray, H. Bulut, and F. B. M. Belgacem, Sumudu Transform Method for Analytical Solutions of Fractional Type Ordinary Differential Equations, Mathematical Problems in Engineering, Article ID 131690. (2015)

10. A. Atangana and A. Kilicman, The use of Sumudu transform for solving certain nonlinear fractional heatlike equations, Abstract Appl. Anal., Vol. 2013, Article ID 737481, 12 pages. (2013)

8. Z. Hammouch, T. Mekkaoui, Traveling-wave solutions of the Generalized Zakharov Equation with time-space fractional derivatives, MESA, 5(4), 489-499. (2014)

\footnotetext{
${ }^{*}$ Corresponding author: gulnuryel33@gmail.com
} 
11. X. F. Yang, Z. C. Deng and Y. Wei, A Riccati Bernoulli sub-ODE method for nonlinear partial differential equations and its application, Adv. Differ. Equ., 117, 1-17. (2015)

12. V.G. Drinfel'd and V.V. Sokolov, Equations of Kortweg-de Vries type and simple lie algebras, Sov. Math. Dokl. 23, 457-462. (1981) 